\title{
EDITORIAL
}

\section{PREVENTABLE CAUSES OF BLINDNESS}

Blindness is defined as correctable distance visual acuity of 20/200 or poorer in the better eye or visual field less than 20 degrees in its widest diameter (1) It is estimated that there are about 15 million blind people on the planet at the moment (1). The leading causes of preventable blindness today are trachoma, onchocerciasis, xerophthalmia and leprosy (1). Other preventable causes include other infections like measles. Malnutrition also plays a major role in causing preventable blindness.

Trauma is also a cause of blindness and this includes physical injury and chemical burns which may occur accidentally or intentionally; both are preventable. latrogenic causes like long-term drug toxicity are recognised and are largely preventable.

The World Health Organisation estimates that more than six million people are blind worldwide from trachoma alone, making it the greatest cause of preventable blindness in the world (1). It is amazing that trachoma has been endemic in many parts of the world since pre-history especially in the Middle East, and there had been devastating outbreaks in the wake of the crusades and Nepoleonic wars but still occupies that unfortunate position as the leading cause of blindness worldwide.

Onchocerciasis results from investation with the nematode onchocerca volvulus, transmitted by a blood sucking fly of the genus simulium. This disease is endemic in Central America and Tropical Africa especially West Africa, The Nile valley in Uganda (hence the name Jinja fly for simulium) (2) and the coastal belt of Kenya.

It is estimated that two million people worldwide are blind from onchocerciasis alone (1). The microfiliaria cause sclerosing keratitis, iritis, chorioretinal degeneration and optic atrophy. The eye changes improve markedly on treatment with diethylcarbamazine.

Xerophthalmia is the leading cause of preventable childhood blindness. It is secondary to vitamin A deficiency. It is estimated that about twenty thousand children go blind world wide from this cause alone every year, and this is probably an underestimation.
In the United States of America, 53\% of the blind are 65 years old or older. Although blindness rate is equal between men and women in the USA, the prevalence of blindness among Afro-Americans is double that of white Americans (1). Different patterns are expected in different regions of the world.

In many parts of the world where people rely on marginal diets and experience frequent food shortages, measles, a viral infection of childhood is a major cause of preventable blindness among children. This is thought to be caused by an acute deficiency of vitamin A. Against this background the viraemia leads to the so-called corneal melting syndrome invariably leading to blindness or severe corneal specifications. In this regard recent resurgence of measles in Kenya does not augur well for children of this land, and the recent severe food shortages are not helping matters either.

Vitamin A deficiency does occur among adult population notably among long-term inmates serving sentences of five years or more. Blindness from this cause is not rare but obviously preventable.

Leprosy is a disease caused by the acid fast bacillus Mycobacterium leprae. The clinical picture is influenced by the immunity of the host, if immunity is high, the paucibacillary type is seen. In this type the eyelids may stiffen making eye closure impossible. Exposure keratitis results which may lead to blindness. If immunity is low the multibacillary form occurs. The organisms invade the uvea causing chronic and progressive uveitis which often leads to phthisis bulbi and blindness.

Varieties of trauma includes military activity, assault, domestic violence and accidents including road traffic accidents. Most of these causes of blindness can be avoided if only mankind would agree to resolve disputes using non-violent techniques. Domestic violence as a cause of blindness is rare (3) although when it occurs it is usually quite dramatic. Cases have been reported in the media of one spouse gouging out the eyes of the other. Pathological jealousy is usually the underlying state of mind. Two women fighting, usually over a man, are known to go for each 
other's faces, eyes included. A report from Nigeria quite clearly demonstrated this as reported in "Post graduate Doctor." In fact bilateral enucleation under such circumstances has been reported (4). In the USA trauma accounts for $4 \%$ of cases of blindness (1).

Chemical burns may occur as a result of accidents or assault. The accidental ones usually occur under industrial settings and involve acids or caustics or may accidentally occur in a beauty saloon during hair grooming.

Assault cases usually involve acts by criminals or jealousy rage. In this latter case hot water is a more common material used to cause intended disfigurement in the Kenyan setting. Avoidance of such acts is not in doubt.

Retino - toxic drug reactions: Although largely iatrogenic in nature drug adverse effects can be monitored using both subjective tests for instance, by visual acuity assessment or objective techniques, such as, ophthalmoscopy or slit-lamp biomicroscopy. Long-term use of drugs like chloroquine in susceptible individuals, including the mentally-ill, may cause severe eye damage leading to blindness. Alternative drugs should be used but are rarely considered for such people.

Neuro-toxicity to optic nerve is well documented when heavy metals are used for treatment, for instance, in cases of syphilis. Heavy smoking combined with diets high in cyanide content such as, cassava and kales (sukuma wiki) may lead to optic nerve damage leading to blindness (5). These can be avoided by excluding these foods from the diet or prompt administration of hydroxycobalamin. Kales belong to a group of plants called brassica which are known to have high cyanide content.

Tuberculosis is becoming more commonly diagnosed now worldwide partly because of HIV /
AIDS pandemic. Anti TB drug, ethambutol, is therefore more frequently used nowadays. This drug is particularly toxic to optic nerve and may cause blindness. This risk had been assessed by Tamai in 1968 as $3.5 \%$ among patients using ethambutol (6). Awareness of this risk is more urgent now than ever before.

Methyl alcohol is a vicious retinal and optic nerve poison. Ingestion is often followed by vomiting, prostration, coma, convulsions and bilateral blindness and death. The so-called "kumi kumi" tragedy in Kenya is well known. Law enforcement agencies need to have a second look at this recurrent and often tragic cause of preventable blindness and death. The root causes of this problem should be sought. The victims invariably go blind before they die. Methyl alcohol is poison and is never meant to be consumed as a drink.

P.J. Onyango-Ogony, MBChB (Mak), DORCS (Lond), MMed (Opthal) (Nbi), Consultant Ophthalmologist, formerly Chief Ophthalmologist and Head of Department of Ophthalmology, Kenyatta National Hospital, P.O. Box 20829, Nairobi.

\section{REFERENCES}

1. Fraunfelder F.T., Roy F. and Jean Grove W.B. Current ocular Therapy, Saunders Co. 1995.

2. P.T. Roper. Lectured Notes on Ophthalmology, $3^{\text {rd }}$ edition.

3. Onyango-Ogony P.J. Ophthalmic aspects of domestic violence in Nairobi, Kenya. A paper read at the annual conference of Ophthalmological Society of East Africa in Kampala, Uganda, 1996.

4. Dr. Mathenge, Personal Communication.

5. Rose C. Medical Ophthalmology, 1976.

6. Tamai A. Jap. J. cl. ophthal. 1968; 221068 quoted by Friedmann A.I. 